\title{
Development of a rapid dipstick with latex immunochromatographic assay (DLIA) for diagnosis of schistosomiasis japonica
}

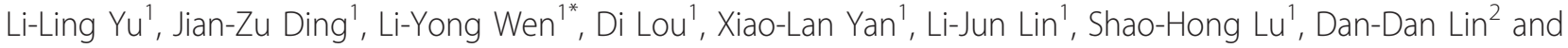 \\ Xiao-Nong Zhou ${ }^{3}$
}

\begin{abstract}
Background: Schistosomiasis japonica (schistosomiasis) is a zoonosis that can seriously affect human health. At present, the immunodiagnostic assays for schistosomiasis detection are time-consuming and require well-trained personnel and special instruments, which can limit their use in the field. Thus, there is a pressing need for a simple and rapid immunoassay to screen patients on a large scale. In this study, we developed a novel rapid dipstick with latex immunochromatographic assay (DLIA) to detect anti-Schisaosoma japonicum antibodies in human serum.
\end{abstract}

Results: Using latex microspheres as a color probe, DLIA was established to test standard positive and negative sera, in comparison with the classical enzyme-linked immunosorbent assay (ELISA). The sensitivity and specificity of DLIA were $95.10 \%$ (97/102) and 94.91\% (261/275), respectively. The cross-reaction rates with clonorchiosis, intestinal nematodes, Angiostrongylus cantonensis and paragonimiasis were 0, 0,0 and $42.11 \%$ respectively. All the results showed no significant difference to the ELISA. In field tests, 333 human serum samples from an endemic area were tested with DLIA, and compared with ELISA and Kato-Katz method. There was no significant difference between DLIA and ELISA on positive and negative rates of detection; however, significant differences existed between DLIA and Kato-Katz method, and between ELISA and Kato-Katz method. The kappa value between DLIA and ELISA was 0.90 .

Conclusions: This is the first study in which DLIA was used to detect anti-Schistosoma japonicum antibody. The results show that DLIA is a simple, rapid, convenient, sensitive and specific assay for the diagnosis of schistosomiasis and is therefore very suitable for large-scale field applications and clinical detection.

\section{Background}

Human schistosomiasis is a zoonosis that has endangered human health for thousands of years [1,2]. A recent report indicates that approximately 200 million people are infected and over 750 million people are at risk of infection [3]. Five species of the Schistosomatidae family are human parasites and, in China, the disease is caused by schistosomiasis japonica (schistosomiasis) [2-5]. It has been endemic for centuries and remains a serious public health problem in China [6]. According to the Chinese annual disease report, there are 365770

\footnotetext{
* Correspondence: wenliyong@126.com

'Institute of Parasitic Diseases, Zhejiang Academy of Medical Sciences, Hangzhou, 310013, China

Full list of author information is available at the end of the article
}

patients and approximately 250 million people are at risk in 2009 [7].

The Schistosoma infection rate has decreased with improvements in disease control, thus, diagnosis is the key to schistosomiasis control. The diagnostic methods include direct parasitological observations (parasite egg detection and miracidium hatching) and indirect serological techniques (antibodies or circulating antigen detection in serum) [4]. Microscopic examination of stool (e. g., Kato-Katz method and miracidium hatching tests) is considered the "gold standard" for the detection of schistosomiasis [8]. However, these procedures are timeconsuming and limited in sensitivity [9-11] due to great day-to-day fluctuations in egg output. Meanwhile, the Kato-Katz method is based on microscopic examination of a $41.7 \mathrm{mg}$ faecal sample, and this quantity is quite

\section{Biomed Central}


small, which can cause high false negative rates. Therefore, the detectable rate is much lower in the Kato-Katz method which depends on the intensity of infection [12]. It had been reported that in a lightly endemic area for schistosomiasis, the disease would not be detected by the Kato-Katz method [13], since the stool sample investigated normally contains less than $2 \%$ of the daily egg output [12]. This situation calls for the development of more sensitive methods to supplement or replace stool examination, because less sensitive diagnostic assays are unsuitable for the evaluation of control programmes, such as morbidity reduction by chemotherapy, which normally leads to a reduction in prevalence and an increase in the number of low intensity infections.

Immunodiagnostic assays are already used in China for selective chemotherapy and for the large-scale screening of target populations. Over the past 10-20 years, the most promising alternative diagnostic methods developed against schistosomiasis are the intradermal test (ID) [14], the circumoval precipitin test (COPT) [15], an indirect hemagglutination assay (IHA) [16], and enzyme-linked immunosorbent assay (ELISA) [17]. These methods have high sensitivity and specificity and have been widely used in China. Nonetheless, these methods have some shortcomings, such as being timeconsuming and requiring special equipment and reagents, which make them unfit for field and bed application. Thus, better and more convenient screening methods are urgently needed for the detection of schistosomiasis.

Immunochromatography assay is a simple, rapid and convenient method. As a type of new diagnostic technology, it was developed after the immunofluorescence assay, ELISA and radioimmunology assay. An immunochromatographic assay was first used during the early 1990s to test for human chorionic gonadotropin (HCG) [18] and then subsequently for the detection of hepatitis B virus surface antigen (HBsAg) [19]. Now, it is widely used in various fields of medicine. In this study, we used latex microspheres as a color probe to establish a novel, simple, rapid and convenient immunoassay with high sensitivity and specificity for the screening of Schistosoma infected persons on a large scale in endemic areas and assess its usefulness for clinical diagnosis. On the basis of the immunochromatographic assay, we labelled mouse anti-human IgG with latex microspheres, and developed a rapid anti-Schistosoma antibody detection dipstick, which we named the dipstick with latex immunochromatography assay (DLIA). Its application has not been reported previously elsewhere. We assessed the diagnostic value of the DLIA in the laboratory and field for detection of anti-Schistosoma antibodies, and compared the results with the ELISA and Kato-Katz method.

\section{Material and methods}

\section{Soluble egg antigen (SEA) of Schistosoma japonicum}

The ova of Schistosoma were collected from liver tissue of artificially infected rabbits. After repeated washing, the purified ova were ground into a fine powder using a pestle and mortar. Subsequently, this powder was diluted with normal saline to make a $1 \%$ SEA solution. After alternate cryorupture at temperatures of $-20^{\circ} \mathrm{C}$ and $37^{\circ} \mathrm{C}$ for three rounds, the solution was kept for three days at $4^{\circ} \mathrm{C}$. The solution was ultrasonicated on ice three times ( 2 min each time). After $30 \mathrm{~min}$ centrifugation at $851 \times \mathrm{g}$ followed by, $20 \mathrm{~min}$ at $7661 \times \mathrm{g}$, a clear liquid with a protein content of $15 \mathrm{mg} / \mathrm{ml}$ resulted and was stored at $-20^{\circ} \mathrm{C}$.

\section{Antibodies for labelling and quality control}

Mouse anti-human IgG was obtained from Shanghai Biology Institute, China, the protein concentration was 5 $\mathrm{mg} / \mathrm{ml}$ and the ELISA titer was 1:50,000. Goat antimouse IgG was obtained from Shanghai Biology Institute, China, the protein concentration was $12 \mathrm{mg} / \mathrm{ml}$ and its agar gel precipitation titer was 1:64. HRP enzyme-labelled goat anti human IgG was produced by Hangzhou Longji Biotechnology Co. Ltd, China.

\section{Procedure of mouse anti -human IgG labelled with latex microspheres}

Red latex particles and borax buffer ( $\mathrm{pH}$ 8.5) were mixed and centrifuged for three minutes at $19000 \times \mathrm{g}$. The liquid supernatant was discarded, meanwhile 4-morpholineethanesu (MES), N-hydroxysuccinimide (NHS) and 1-ethyl-3-(3-dimethyllaminopropyl) carbodiimide hydrochloride (EDC HCL) was added into the precipitate. After $30 \mathrm{~min}$, the solution was centrifuged for $30 \mathrm{~min}$ at $19,000 \times \mathrm{g}$ before the liquid supernatant was discarded again. Next, the precipitate was dissolved in borax buffer ( $\mathrm{pH} 8.5$ ), added mouse anti-human IgG into the solution, and shaking for $30 \mathrm{~min}$ and then centrifuged for $30 \mathrm{~min}$ at $19000 \times \mathrm{g}$. The liquid supernatant was discarded, and the precipitate was blocked with casein blocking buffer. The final concentration of mouse anti-human IgG was $1 \mathrm{mg} / \mathrm{ml}$. The color probe was kept at $4^{\circ} \mathrm{C}$. All chemicals were purchased from Hangzhou Longji Biotechnology Co. Ltd, China.

\section{DLIA dipstick preparation}

The dipsticks were assembled on a PVC pad (40 $\mathrm{mm} \times 300 \mathrm{~mm})$. The dipstick consisted of a waterabsorbing pad, an application pad, a sample adsorbing pad and an NC membrane. On the top of the PVC pad, a paper pad (Biotechnology Shanghai Co. Ltd., China) was attached as the water-absorbing pad. At the bottom of the dipstick, glass fibers (Biotechnology Shanghai Co. 
Ltd) were attached as the sample absorbing pad. The application pad was attached next to the sample pad, which was made of glass fiber membrane (Biotechnology Shanghai Co. Ltd) for conjugated latex microspheres (200 nm, red; Biotechnology Shanghai Co. Ltd) labelled with mouse anti-human IgG. The NC membrane (Millipore SHF 135, USA) was attached between the application pad and the water-absorbing pad and was used as an adsorption pad. SEA was attached on the test line and goat anti-mouse IgG was attached on the control line. The SEA and goat anti-mouse IgG were jet-positioned on to the NC membrane pad at a distance of 4 $\mathrm{mm}$. After all the pads were assembled on the PVC backing, the combined strips were cut to dipsticks measuring $4 \mathrm{~mm} \times 40 \mathrm{~mm}$. The schematic of dipstick is shown in Figure 1.

The optimal conditions for the DLIA dipstick assembly adjustment including the conditions as follows: the concentration and the volume of SEA on the test line, the concentration and the volume of goat anti-mouse IgG on the control line, the dilution proportion of latex conjugate, the volume of serum used for testing, reaction time, and so on. A first step of the process of adjustment, involved several different concentration of SEA (such as $0.04,0.08,0.16,0.32 \mathrm{mg} / \mathrm{ml}$ respectively) sprayed on the test line, meanwhile, the other conditions of the dipstick was under the assumption value. Next, the dipstick was assembled and tested with the positive and negative standard serum respectively. Then, we compared the sensitivity, specificity and dipstick background, and chose the best concentration of SEA. The others conditions were tested in a similar manner, and finally, we obtained the optimal conditions (data not shown).

\section{DLIA procedure}

Analysis in sera samples was done by adding $50 \mu \mathrm{l}$ serum on to the sample absorbing pad well. The reaction of the anti-SEA antibody and mouse anti-human IgG labelled with latex microspheres took place immediately at the conjugate pad area. Then, the compound of anti-SEA antibody with latex colour probe was combined with SEA which sprayed on the test line if there was anti-SEA in the serum. If anti-SEA was absent, no reaction would occur. The rest of the conjugate then passed though the test line and bind with goat antimouse IgG at the control line. The intensity of color developed due to the presence of tracer and this correlate with the amount of anti-SEA antibody present in the sample. If the test line and control line were colored red, the sample was recorded as positive. If only the control lined was colored red but the test line was not colored, the test result was negative. If neither of the lines on the dipstick were colored, the test reagents were assumed not to be working and the test had to be repeated. The result of dipstick latex particle immunoassay for schistosomiasis detection is shown in Figure 2.

In all these experiments, the results must be read at 15 min after the sample added. Some development on the test line was noted after $15 \mathrm{~min}$, and the trace of any line should be held void.

\section{Serum samples}

102 standard positive samples for schistosomiasis were parasite positive by the Kato-Katz method [eggs per gram (EPG): 8 1352]. In total, 275 standard negative samples for schistosomiasis were obtained from healthy people. The 15 serum samples with clonorchiosis, 19 serum samples with paragonimiasis, 11 serum samples

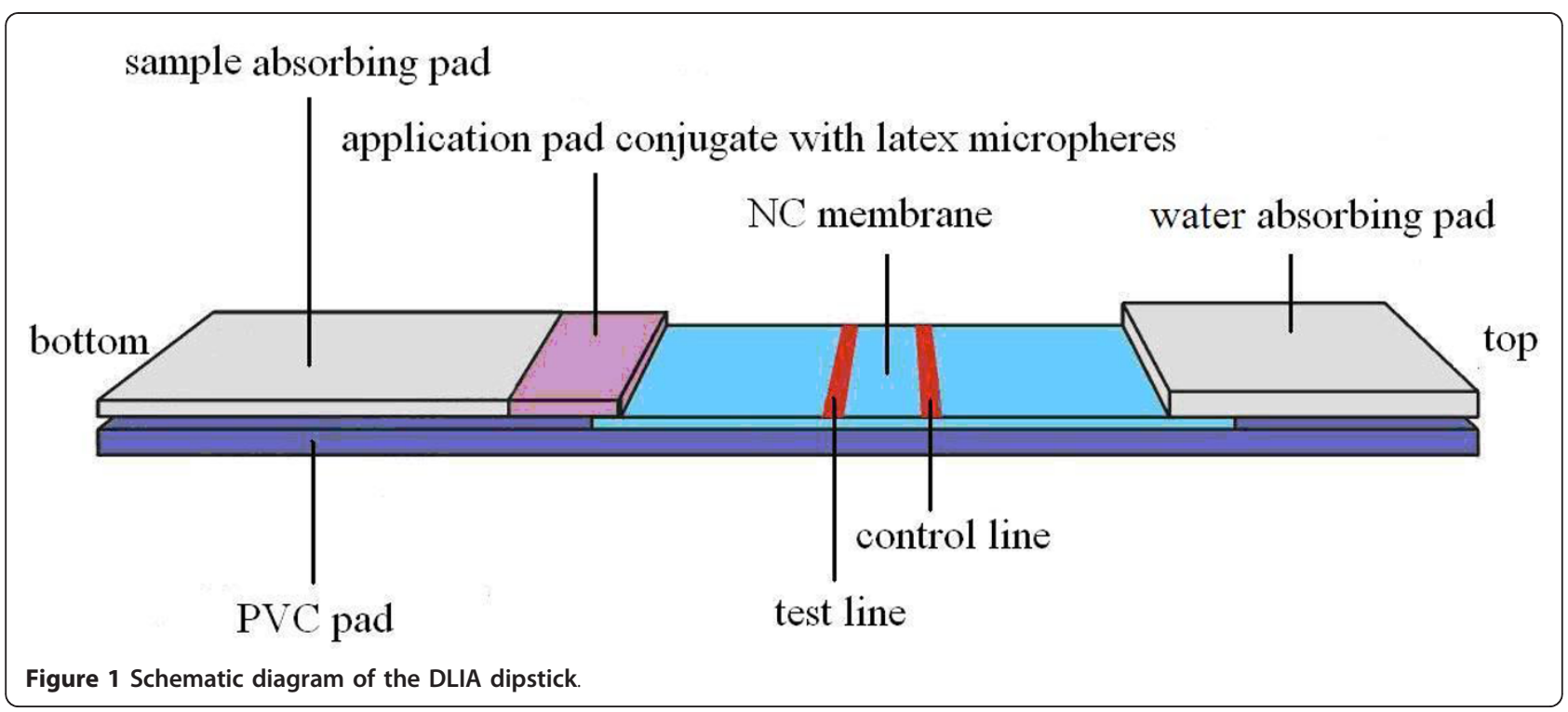




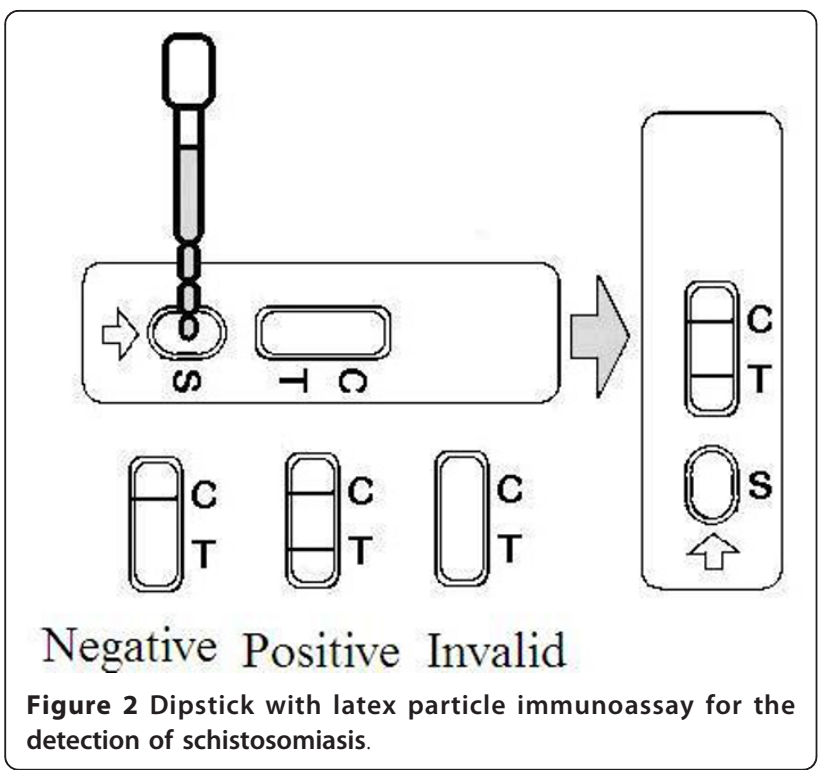

with intestinal nemathelminthiasis, 8 serum samples with Angiostrongylus cantonensis(all sera provided by Zhejiang Academy of Medical Sciences, China). The sera were stored in aliquots at $-20^{\circ} \mathrm{C}$.

\section{Field application}

A village in a Schistosoma-endemic area (Jiangxi Province, China) was selected as a study site. The 333 persons from 6-65 years old in the village were randomly collected to offered serum samples and stool samples. All the serum samples were tested by DLIA and ELISA respectively. And all the faecal samples were tested only by Kato-Katz method.

\section{ELISA}

SEA antigen $(120 \mu \mathrm{l}, 10 \mu \mathrm{g} / \mathrm{ml})$ was added into the ELISA plates and incubated at $4^{\circ} \mathrm{C}$ overnight. The plate was washed with phosphate-buffered saline (PBS) before the microwells were blocked with blocking solution. Then, $100 \mu \mathrm{l}$ of diluted human serum (1:200) was added and the microwells incubated at $37^{\circ} \mathrm{C}$ for $30 \mathrm{~min}$. The plate was washed again and $100 \mu \mathrm{l}$ of horseradish peroxidase -labelled goat anti-human IgG (1:2000 diluted) was added and incubated at $37^{\circ} \mathrm{C}$ for $30 \mathrm{~min}$. After washing, the substrate 3,3-,5,5-tetramethyl benzidiny (TMB) was added into each well. The reaction was stopped by adding $50 \mu \mathrm{l}$ of $\mathrm{H}_{2} \mathrm{SO}_{4}(2 \mathrm{~mol} / \mathrm{l})$. Absorbance was measured at $450 \mathrm{~nm}$ by an ELX800 multilabel plate reader (Biotek, USA).

\section{Kato-Katz method}

All the faecal samples were tested only by Kato-Katz method. The procedure of the Kato-Katz method for detection of Schistosoma eggs was conducted as previously described [13]. Stool samples were processed following the procedure of Kato-Katz method. Three slides, each containing $41.7 \mathrm{mg}$ of sieved stool were made from each stool specimen by qualified technicians and were examined in a blinded manner. The number of eggs in each slide was counted and recorded. The infection intensity of each individual was recorded as EPG (eggs per gram feces) calculated by summing the results of three slides and multiplying by 8 .

\section{Youden index}

Youden index $=$ sensitivity + specificity -1, ranged from 0 to 1.

The Youden Index is used for evaluate the authenticity of the test, which means the capability of the screening test method to distinguish between the real patients and healthy people. The higher the Youden Index of the method, the greater authenticity of the screening test, and the better of the diagnostic kit.

\section{Stability of DLIA}

The DLIA dipsticks were stored at $4{ }^{\circ} \mathrm{C}, 25^{\circ} \mathrm{C}$ and $37^{\circ} \mathrm{C}$, and tested for sensitivity and specificity of the dipsticks by positive and negative standard serum each week.

Three batches of latex conjugated microspheres were synthesized and assembled into dipsticks. All the dipsticks were tested with sensitivity and specificity by positive and negative standard serum.

\section{Ethical considerations}

Written ethical approval for this study was obtained at the Ethics Committee of Zhejiang Academy of Medical Sciences, China.

Written informed consent was obtained from all subjects and from parents or guardians of minors who were involved in the project. Study participants identified as positive for schistosomiasis were treated with $40 \mathrm{mg} / \mathrm{kg}$ praziquantel.

\section{Statistical analysis}

The SPSS statistical package for social sciences, version 11.5 software (SPSS Inc., Chicago, IL, 2002) was used for statistical analysis. In the field application of DLIA, only data from individuals who had provided stool specimen were included in the final analysis.

\section{Results}

\section{Development of DLIA}

DLIA was established using SEA as the target antigen on the test line, with goat anti-mouse IgG as the second antibody on the control line. Red latex labelled with mouse anti-human IgG was used as the color probe. To compare the sensitivity, specificity and dipstick background, the optimal conditions for the DLIA dipstick assembly were determined to be as follows: (1) the concentration of SEA 
was $0.16 \mathrm{mg} / \mathrm{ml}$ and the volume of application was $1.0 \mu \mathrm{l} /$ $\mathrm{cm}$; (2) the concentration of mouse anti-human IgG was $2.0 \mathrm{mg} / \mathrm{ml}$ and the volume application was $1.0 \mu \mathrm{l} / \mathrm{cm}$; (3) the dilution proportion of latex conjugate was $1: 6$ and the volume application was $2.0 \mu \mathrm{l} / \mathrm{cm}$; (4) the volume of serum used for testing was $50 \mu \mathrm{l}$; and (5) the test result was judged after $15 \mathrm{~min}$ by direct observation. The schematic of dipstick is shown in Figure 1.

\section{Sensitivity}

102 serum samples that had been shown to be positive for the Schistosomiasis parasite by the Kato-Katz method (EPG: 8 1352) were used to test the sensitivity of DLIA. ELISA was also performed on these samples for additional comparison. The sensitivity of DLIA and ELISA were $95.10 \%$ (97/102) (Table 1). There was no difference in sensitivity between these two assays.

\section{Specificity}

Overall, 275 serum samples from healthy individuals (i. e., negative for schistosomiasis on the Kato-Katz method) were used to assess the specificity of DLIA and ELISA. The specificity of DLIA and ELISA were 94.91\% $(261 / 275)$ and $97.45 \%(268 / 275)$, respectively (Table 1$)$. There was no significant difference in specificity between the two assays $\left(\chi^{2}=2.43, P>0.05\right)$.

\section{Youden index}

Youden index of DLIA and ELISA were 0.90 and 0.93 for schistosomiasis diagnosis, respectively.

\section{Cross-reactivity}

Fifteen clonorchiosis, 11 intestinal nematode and 8 Angiostrongylus cantonensis samples were tested with DLIA and ELISA, and showed no signs of cross-reactivity (Table 1). When 19 paragonimiasis samples were tested by DLIA and ELISA, the cross reaction rates were $42.11 \%(8 / 19)$ and $47.37 \%(9 / 19)$, respectively (Table 1$)$, but there was no significant difference in the cross-reactivity between the two assays $\left(\chi^{2}=0.11, P>0.05\right)$.

\section{Stability of DLIA}

The DLIA dipstick was stored for 1 year at various temperatures (i.e., $4^{\circ} \mathrm{C}, 25^{\circ} \mathrm{C}$ and $37^{\circ} \mathrm{C}$ ) without loss of activity (data not shown). Reconstituted detection reagent could be stored at $4^{\circ} \mathrm{C}, 25^{\circ} \mathrm{C}$, and $37^{\circ} \mathrm{C}$ for at least 1 year. Three batches of latex conjugated microspheres were tested after assembly into dipsticks and these results showed that the dipsticks were stable in each batch (data not shown).

\section{Field application of DLIA}

Serum samples (333 in total) from local people in an area endemic for schistosomiasis were tested by DLIA and ELISA. Fecal samples from these people were only tested by the Kato-Katz method. The positive rates by DLIA and ELISA were 21.62\% (72/333) and 24.02\% (80/ $333)$, respectively. There was no significant difference in positive detection rate between DLIA and ELISA $\left(\chi^{2}=\right.$ $0.55, P>0.05)$. Seventy samples were positive and 251 were negative in both the DLIA and ELISA tests. Two cases tested positive by DLIA but were negative by ELISA, and 10 cases that tested positive by ELISA were negative by DLIA. The DLIA results are compared with ELISA, there was $96.40 \%$ agreement $(321 / 333)$. The kappa value between DLIA and ELISA method was 0.90, which indicated that the observed value of DLIA had mid-range coincidence with ELISA (Table 2).

Stool examination conducted for all 333 persons by Kato-Katz method gave a positive rate of $3.90 \%$ (13/ 333). There was significant difference in positive rate between DLIA and Kato-Katz method $\left(\chi^{2}=46.94, p<\right.$ $0.05)$. Both assays recorded positive detection in 12 samples, while the tests were both negative in another 260 samples. A further 60 cases tested positive by DLIA but negative by Kato-Katz method, and only one case tested positive by Kato-Katz method but was negative in the DLIA Assay (Table 3).

\section{Discussion}

In this study, DLIA was used for the detection of antiSchistisoma antibodies in human sera at first time and the results showed a $95.10 \%(97 / 102)$ sensitivity and a $94.91 \%(261 / 275)$ specificity for detection. The crossreaction rates with clonorchiosis, intestinal nematodes, Angiostrongylus cantonensis and paragonimiasis were 0 (0/15), $0(0 / 11), 0(0 / 8)$ and $42.11 \%(8 / 19)$, respectively. Compared with ELISA (the classic diagnosis method), the results showed that no significant differences existed between the two methods. Youden index of DLIA and ELISA were 0.90 and 0.93 for schistosomiasis diagnosis, respectively.

Importantly, in addition to being rapid and simple to use, the new DLIA technique does not require any specific instruments and can be stored at $4^{\circ} \mathrm{C}, 25^{\circ} \mathrm{C}$ and $37^{\circ}$ $\mathrm{C}$ for at least one year. Besides this, the DLIA dipstick could be easily delivered anywhere, so, it is very suitable for rapid diagnosis and large scale applications in the field.

Currently, a few immunochromatographic assay technologies have been used for the diagnosis of schistosomiasis japonica, such as the dipstick dye immunoassay (DDIA) [20] and the colloidal gold immunochromatographic assay (GICA) [21]. DIGFA as a rapid serological test method was based on immunofiltration assay and applied in China for several years. DIGFA and GICA use colloidal gold as the color probe, while DDIA uses a colloidal dye as the color probe. Compared with these 
Table 1 Serum detection of schistosomiasis by DLIA and ELISA

\begin{tabular}{cccccc}
\hline Group & Number of sera & \multicolumn{2}{c}{ DLIA } & ELISA \\
\cline { 3 - 6 } & & Number positive & Positive detection rate (\%) & Number positive & Positive detection rate (\%) \\
\hline Schistosomiasis & 102 & 97 & 95.10 & 9.09 & 95.10 \\
Normal individuals & 275 & 14 & 0 & 7 & 2.55 \\
Clonorchiosis & 15 & 0 & 42.11 & 0 & 0 \\
Paragonimiasis & 19 & 8 & 0 & 9 & 47.37 \\
Intestinal nematodes & 11 & 0 & 0 & 0 & 0 \\
Angiostrongylus cantonensis & 8 & 0 & 0 & 0 \\
\hline
\end{tabular}

kits, DLIA has higher sensitivity and specificity than GICA [21], a lower cross-reaction rate than DDIA (42\% compared with $70 \%$ for DDIA) [20], and is more convenient to use than DIGFA. The DLIA uses latex microspheres as color probes. Latex microspheres are a type of polymer that binds to particles and disperses throughout a fluid but poses no threat as an environmental pollutant. The carboxyl group of the latex microspheres is activated and binds to the amino-group on the protein. This chemical bond is stable and is not affected by changes in $\mathrm{pH}$, salinity and ionic strength of the environment, which makes it convenient to store for a long time. As a marking agent, the diameter of latex particles is $200 \mathrm{~nm}$, which means results will show up clearly with very tiny amounts of antigen or antibodylabel bound to it. In contrast, the diameter of colloidal gold is much smaller than latex particles that means much more antigen or antibody is needed during the labelling step otherwise the result on the nitrocellulose (NC) membrane will not be clear enough to read. The test area on the dipstick used colloidal gold as color probe which could show more clearly with either a higher concentration of antigen or antibody, or with a larger diameter of immunogold (e.g., 40 50 nm). However, although larger colloidal gold increases the sensitivity of the test, the colloidal gold conjugate would be less stable and would precipitate easily. Moreover, the process of immunogold probe labelling would be affected by changes in $\mathrm{pH}$, salinity and ionic strength of the milieu. Furthermore, due to the physical combination between immunogold and protein [22], it is easily dissociated by changes in $\mathrm{pH}$, salinity and ionic strength. So, latex microspheres have several advantages as a color probe compared with colloidal gold. So, as the results in this study proved, the dipstick based on latex microspheres has a higher sensitivity than that based on colloidal gold. As a color probe, latex microspheres can be kept at $4^{\circ} \mathrm{C}-37^{\circ} \mathrm{C}$ for at least 1 year, which is much longer than that of colloidal gold and colloidal dye $[20,21]$. Despite the cross-reaction rate of paragonimiasis by DLIA was $42 \%$, which is much lower than the $70 \%$ cross-reactivity of DDIA [20], this is still a concern with the dipstick method. Reducing this cross-reactivity will be addressed in further studies.

As a classic method, ELISA is currently the most sensitive method for schistosomiasis diagnosis [23]. As a rapid and convenient schistosomiasis screening test method, DLIA was used in a field test on 333 human sera samples and compared with ELISA. The results of the tests showed that there were no significant differences in positive and negative detection rates between the two methods. According to Feiss's theory, a kappa value of $0.75 \sim 1.00$ means well-matched, $0.4 \sim 0.74$ means a distinct correlation and 0.01 0.39 means no match. Thus, the kappa value of field application was 0.90, which means that DLIA has the same ability as ELISA for identifying patients with or without schistosomiasis. However, ELISA reagents require cold-chain logistics and the test takes several hours to complete. Another advantage of DLIA is cost, as the latex microspheres produced in China are considerably less expensive than enzyme conjugates or colloidal gold. In addition, the control band on each dipstick contributes to the quality control of the assay.

However, microscopic examination of stool (e.g., KatoKatz method and miracidium hatching tests) is still considered the "gold standard" for the detection of schistosomiasis. In this research, the positive rate of stool examination was much lower than DLIA and ELISA. This could be explained by one or more of the following

Table 2 Detection of schistosomiasis during the field application of DLIA and ELISA

\begin{tabular}{cccc}
\hline DLIA & ELISA & Total number of samples \\
\cline { 2 - 4 } & Number positive & Number negative & \\
\hline Number positive & 70 & 2 & 72 \\
Number negative & 10 & 251 & 261 \\
Total & 80 & 253 & 333 \\
\hline
\end{tabular}


Table 3 Detection of schistosomiasis during the field application of DLIA and the Kato-Katz method

\begin{tabular}{cccc}
\hline DLIA & \multicolumn{2}{c}{ Kato-Katz } & Total \\
\cline { 2 - 3 } & Number positive & Number negative & \\
\hline Number positive & 12 & 60 & 72 \\
Number negative & 1 & 260 & 261 \\
Total & 13 & 320 & 333 \\
\hline
\end{tabular}

reasons. Firstly, the false negative rate of Kato-Katz method is very high in light epidemic areas. The low sensitivity ranging from $20 \%$ to $70 \%$ for diagnosis of Schistosoma infection [24-26]. Previous research showed that $50 \%$ of the patients in a schistosomiasis endemic area where infection rate is less than $25 \%$ Schistosoma fail to be detected by Kato-Katz method [17]. Furthermore, as the infection rate of schistosomiasis becomes lower [27], it is more and more difficult to identify patients correctly by the Kato-Katz method. Secondly, a recent infection may not have allowed sufficient time after chemotherapy for the antibody level to decline to a normal level, as the anti-Schistosoma antibody in the serum would take months or even years to normal level after treatment [28-31]. And because of it, at present, all the serologic diagnosis methods for schistosomiasis which used to observe the success of treatment were only conducted in the laboratory, but not practiced in the field. Until this time, it is not possible to expect that the existing immunodiagnostic approach can meet the requirements for control programs of schistosomiasis and the DLIA, like the other serologic diagnosis methods, was unfit for observe the success of treatment. Thirdly, cross-reactions with other parasitic infections can cause false positive results. Notably, $\mathrm{He}$ et al. reported that it was not entirely correct to judge the reliability of immunodiagnostic methods by the results of stool examination [32].

According to the results of the test, the DLIA dipstick could be stored at $4{ }^{\circ} \mathrm{C} \sim 37^{\circ} \mathrm{C}$ for at least one year, and the test could be finished in 15 min without any instrument or reagents. So, it could be easily delivered anywhere, and very suitable for rapid diagnosis and largescale applications in the field.

\section{Conclusions}

The present study is the first report to use latex microspheres as a color probe to test for schistosomiasis. The results of the research showed that the DLIA assay has the same ability as ELISA to identify schistosomiasis patients and to show that the DLIA has high sensitivity and specificity with no cross-reaction with clonorchiosis, intestinal nematodes and Angiostrongylus cantonensis. In summary, DLIA provides a rapid, simple, convenient, easy to carry and reliable diagnosis for schistosomiasis detection, especially for screening patients in a largescale or for fast clinical diagnosis in the laboratory. Further studies are required to reduce the cross-reaction rate and focus on developing a semi-quantitative or fully quantitative method. In the future, we also planned to develop a series of dipstick based on the immunochromatography assay to test for paragonimiasis, toxoplasmosis and other infections.

\section{Acknowledgements}

This work is financially supported by the National S \& T Major Program (Grant No. 2008ZX10004-011), the Projects of Zhejiang Health Department (Grant No. XKQ-009-003, XKQ-010-001, 2010KYA001), the Projects of Zhejiang Scientific and Technological Department (Grant No. 2007F30016, 2008F1031,2009F20036) and Zhejiang Provincial Program for the Cultivation of High-level Innovative Health Talents.

\section{Author details}

'Institute of Parasitic Diseases, Zhejiang Academy of Medical Sciences, Hangzhou, 310013, China. ${ }^{2}$ Jiangxi Provincial Institute of Parasitic Diseases, Nangchang, 330046, China. ${ }^{3}$ National Institute of Parasitic Diseases, Chinese Center for Disease Control and Prevention, Shanghai, 200025, China.

\section{Authors' contributions}

LY and JD developed the DLIA dipstick, LY, JD and DL contributed to sensitivity, specificity, cross-reaction, LY, DL and XZ contributed to field application detection. LL, XY and SL established the test data bank and analyzed the test results. Liyong Wen created the detailed experimental design. All authors read and approved the final version of the manuscript.

\section{Competing interests}

The authors declare that they have no competing interests.

Received: 18 March 2011 Accepted: 9 August 2011

Published: 9 August 2011

\section{References}

1. David RC: Schistosomiasis: overview of the history, biology, clinicopathology, and laboratory diagnosis. Clin Microbiol News/2005, 27:163-169.

2. Gryseels B, Polman K, Clerinx J, Kestens L: Human schistosomiasis. Lancet 2006, 368:1106-1118.

3. Steinmann P, Keiser J, Bos R, Tanner M, Utzinger J: Schistosomiasis and water resources development: systematic review, meta-analysis, and estimates of people at risk. Lancet Infect Dis 2006, 6:411-425.

4. Shazly SE, Saadany SE: Schistosomiasis. Tanta Med Sci J 2006, 1:1-10.

5. Borch M, Kiernan M, Rust K, Baron B, Simmons B, Hattala P, Davey A, Yovanovich J, Shayder D, Wasilewski A, LaFaro VE: Schistosomiasis: a case study. Urol Nurs 2009, 29:26-29.

6. Zhu R, Darren JG, Trift AP, Williams GM, Yi Z, Qiu DC, Zheng F, Li YS, Guo J, Zhu HQ, Wu WP, Li RS, McManus DP: A 5-year longitudinal study of schistosomiasis transmission in Shian village, the Anning River Valley, Sichuan Province, the Peoples' Republic of China. Parasit Vectors 2011, 43.

7. Yang H, Hao Z, Rong Z, Gang GJ, Ying WL, Zhao C, Nnong ZX: Schistosomiasis situation in People's Republic of China in 2009. Chin J Schisto Control 2010, 22:521-527.

8. Feldmeier $\mathrm{H}$, Poggensee G: Diagnostic techniques in schistosomiasis control. A review. Acta Trop 1993, 52:205-220.

9. Engels D, Sinzinkayo E, Gryseels B: Day-to-day egg count fluctuation in Schistosoma mansoni infection and its operational implications. Am J Trop Med Hyg 1996, 54:319-324.

10. Yu JM, de Vlas SJ, Yuan HC, Gryseels B: Variations in fecal Schistosoma japonicum egg counts. Am J Trop Med Hyg 1998, 59:370-375.

11. Utzinger J, Booth $M, N^{\prime}$ Goran EK, Muller I, Tanner M, Lengeler C: Relative contribution of day-to-day and intra-specimen variation in faecal egg counts of Schistosoma mansoni before and after treatment with praziquantel. Parasitology 2001, 122:537-544. 
12. Bergquist NR: Present aspects of immunodiagnosis of schistosomiasis. Mem Inst Oswaldo Cruz 1992, 87(Suppl 4):29-38.

13. Katz N, Chaves A, Pellegrino J: A simple device for quantitative stool thick-smear technique in Schistosomiasis mansoni. Rev Inst Med Trop Sao Paulo 1972, 14:397-400.

14. Li Y: Advance of the study on immunodiagnosis of schistosomiasis Nanjing, China: Jiangsu Science Technology; 1991.

15. Zhu YC: Immunodiagnosis and its role in schistosomiasis control in China: a review. Acta Trop 2005, 96:130-136.

16. Hamilton JV, Klinkert M, Doenhoff MJ: Diagnosis of schistosomiasis: antibody detection, with notes on parasitological and antigen detection methods. Parasitology 1998, 117(Suppl):S41-57.

17. Doenhoff MJ, Chiodini PL, Hamilton JV: Specific and sensitive diagnosis of schistosome infection: can it be done with antibodies? Trends Parasitol 2004, 20:35-39.

18. Beggs M, Novotny M, Sampedro S: A selfperforming chromatographic immunoassay for the qualitative determination of human chorionic gonadotrophin(HCG) in urine and serum. Clin Chem 1990, 36:1084-1085.

19. Sumathy S, Thyagarajan SP, Latif R, Madanagopalan N, Raguram K, Rajasambandam P, Gowans E: A dipstick immunobinding enzyme-linked immunosorbent assay for serodiagnosis of hepatitis $B$ and delta virus infections. J Virol Methods 1992, 38:145-152.

20. Zhu Y, He W, Liang Y, Xu M, Yu C, Hua W, Chao G: Development of a rapid, simple dipstick dye immunoassay for schistosomiasis diagnosis. J Immunol Methods 2002, 266:1-5.

21. Zeng XJ, Chen HG, Yuan JH, Jiang WS, Yi FY, Ge J, Hang CQ, Chen ZY, Xie SY: Establishment and evaluation of rapid colloidal gold immunochromatographic assay (GICA) for schistosomiasis diagnosis. Chin J Schisto Control 2005, 17:24-27.

22. Wen LY, Chen JH, Ding JZ, Zhang JF, Lu SH, Yu LL, Shen LY, Wu GL, Zhou XN, Zheng J: Evaluation on the applied value of the dot immunogold filtration assay (DIGFA) for rapid detection of antiSchistosoma japonicum antibody. Acta Trop 2005, 96:142-147.

23. Ross AG, Sleigh AC, Li Y, Davis GM, Williams GM, Jiang Z, Feng Z, McManus DP: Schistosomiasis in the People's Republic of China: prospects and challenges for the 21st century. Clin Microbiol Rev 2001 14:270-295.

24. Zhu HQ, Cao CL, Gao FH, Guo JG, Bao ZP, Wang XH, Dang H, Zhou XN: Evaluation of effectiveness of modified Kato-Katz technique for diagnosis of schistosomiasis japonica. Chin J Schisto Control 2005, 17:273-277.

25. Wu GL: A historical perspective on the immunodiagnosis of schistosomiasis in China. Acta Trop 2002, 8:193-198.

26. Wang $\mathrm{XH}, \mathrm{Wu} \mathrm{XH}$, Zhou XN: Bayesian estimation of community prevalences of Schistosoma japonicum infection in China. Int J Parasitol 2006 36:895-902.

27. Zhou SL, Lin JY, Jiang MS: Schistosoma medicine. 2 edition. Beijing, China: Science Tech Publishing House; 2001.

28. Zhou $X N$, Wang $L Y$, Chen $M G, W u X H$, Jiang $Q W$, Chen $X Y$, Zheng J, Utzinger J: The public health significance and control of schistosomiasis in China-then and now. Acta Trop 2005, 96:97-105.

29. Wu GL: A historical perspective on the immunodiagnosis of schistosomiasis in China. Acta Trop 2002, 82:193-198.

30. Shen L, Zhang ZS, Wu HW, Weir RE, Xie ZW, Hu LS, Chen SZ, Ji MJ, Su C, Zhang $Y$, et al: Down-regulation of specific antigen-driven cytokine production in a population with endemic Schistosoma japonicum infection. Clin Exp Immunol 2002, 129:339-345.

31. Lin DD, Xu JM, Zhang YY, Liu YM, Hu F, Xu XL, Li JY, Gao ZL, Wu HW, Kurtis J, Wu GL: Evaluation of IgG-ELISA for the diagnosis of Schistosoma japonicum in a highprevalence, low intensity endemic area of China. Acta Tropica 2008, 107:128-133.

32. He W, Zhu YC, Liang YS, Dai JR, Xu M, Tang JX, Cao GQ, Hua WQ, Li YL, Yang Z: Comparison of stool examination and immunodiagnosis for schistosomiasis. Chin J Schisto Control 2007, 19:107-109.

doi:10.1186/1756-3305-4-157

Cite this article as: Yu et al.: Development of a rapid dipstick with latex immunochromatographic assay (DLIA) for diagnosis of schistosomiasis japonica. Parasites \& Vectors 2011 4:157.

\section{Submit your next manuscript to BioMed Central and take full advantage of:}

- Convenient online submission

- Thorough peer review

- No space constraints or color figure charges

- Immediate publication on acceptance

- Inclusion in PubMed, CAS, Scopus and Google Scholar

- Research which is freely available for redistribution 